\title{
Complicações clínicas do traumatismo raquimedular: pulmonares, cardiovasculares, geniturinárias e gastrintestinais
}

\author{
Carlos Umberto Pereira ${ }^{1}$, Luciana Franco do Prado de Carvalho ${ }^{2}$, Egmond Alves Silva Santos ${ }^{3}$
}

Universidade Federal de Sergipe (UFS), Aracaju, SE.

Serviço de Neurocirurgia do Hospital João Alves Filho, Aracaju, SE.

Serviço de Neurocirurgia do Conjunto Hospitalar do Mandaqui, São Paulo, SP.

\begin{abstract}
RESUMO
O trauma raquimedular é uma das principais causas de morte entre a população jovem. Além disso, várias complicações são devidas a déficits neurológicos secundários ao trauma raquimedular. A principal complicação é a pulmonar, com alto risco de óbito. A causa mais importante de óbito entre os pacientes com trauma raquimedular é a pneumonia. Entretanto, outras complicações podem estar presentes. Os autores revisaram as complicações clínicas em pacientes com trauma raquimedular.
\end{abstract}

\section{PALAVRA-CHAVE}

Traumatismo raquimedular.

\section{ABSTRACT}

Medical complications in traumatic spinal cord injury: pulmonary, cardiovascular, genitourinary and gastrointestinal

The traumatic spinal injury is one of the most important causes of death in young people. Additionally, many complications are due to neurological deficits secondary to spinal cord injury. The major complication is pulmonary with high risk of death. The most important cause of death among patients with spinal injury is pneumonia. However, others complications can be present. The authors reviewed the clinical complications in patients with spinal injury.

\section{KEY WORD}

Traumatic spinal cord injury.

\section{Introdução}

O traumatismo raquimedular (TRM) resulta de lesões por forças mecânicas aplicadas à coluna espinhal, de intensidade suficiente para lesar o canal vertebral. ${ }^{11}$ Acomete preferencialmente a coluna cervical em 56\% dos casos, seguida por lesões na transição toracolombar (T11-L2) em 24\% dos casos e lombar em 20\% dos casos. ${ }^{24,88,93} \mathrm{O}$ traumatismo craniencefálico (TCE) aparece em $25 \%$ a $50 \%$ dos pacientes com TRM, enquanto $5 \%$ a 10\% dos TCE apresentam TRM associado. ${ }^{44,65}$

Acomete, principalmente, o gênero masculino ${ }^{15,24,26,88}$, na proporção de 4:1, predominando entre 15 e $40 \operatorname{anos}^{24,26}$ ou acima dos 60 anos de idade. ${ }^{24} \mathrm{No}$ Brasil, foram relatados entre 40 a 50 casos novos de TRM/1.000.000 habitantes, aproximadamente o mesmo número de casos relatados nos Estados Unidos. ${ }^{24,26} \mathrm{Na}$ Alemanha, estima-se que, anualmente, haja 17 casos novos/1.000.000 habitantes por ano. ${ }^{26}$ As principais causas descritas de TRM têm sido: acidentes automobilísticos, queda de altura, mergulho em água rasa, ferimentos por arma de fogo e acidentes de trabalho. ${ }^{15,24,26,88}$ Ao exame neurológico, 50\% a 74\% dos pacientes apresentam algum déficit neurológico. ${ }^{33,34}$ A mortalidade é de aproximadamente $50 \%{ }^{48}$ Dezesseis a trinta por cento dos pacientes evoluem para óbito antes da admissão hospitalar ${ }^{92}$ e aproximadamente $20 \%$ dos sobreviventes evoluem para óbito dentro de três meses após o trauma. ${ }^{23,25}$

1. Professor doutor adjunto do Departamento de Medicina da Universidade Federal de Sergipe (UFS), neurocirurgião do Serviço de Neurocirurgia do Hospital João Alves Filho, Aracaju, SE.

2. Doutoranda de Medicina da UFS, Aracaju, SE.

3. Neurocirurgião do Serviço de Neurocirurgia do Conjunto Hospitalar do Mandaqui, São Paulo, SP. 
O nível da lesão neurológica na medula espinhal é classificado com relação ao segmento mais caudal, que apresenta as funções sensitiva e motora preservadas em ambos os lados. ${ }^{26,58,59}$ Geralmente, após traumas severos, pacientes com lesões parciais poderão desenvolver os mesmos sintomas do trauma completo devido ao choque espinhal, retornando às suas funções em direção caudal-rostral nas primeiras 24 horas após a lesão. ${ }^{58,59}$ Em contrapartida, lesões secundárias como edema e hemorragia podem causar deterioração neurológica ascendente..$^{57,59}$

Entre as complicações clínicas gerais, as principais acometem os sistemas respiratório, cardiovascular, gastrintestinal e geniturinário..$^{59,74}$

O presente trabalho tem como objetivo revisar as principais complicações clínicas ocorridas em pacientes vítimas de TRM.

\section{Alterações pulmonares}

As complicações pulmonares são responsáveis pelo maior número de óbito em pacientes com TRM. ${ }^{11,12,20,27,63,81,86,88}$ Sua incidência varia de 36\% a $83 \% .{ }^{12}$ Oitenta por cento dos óbitos em pacientes hospitalizados com lesão cervical ocorrem por causa pulmonar, sendo a pneumonia responsável por $50 \%$ deles. $^{12}$

As complicações pulmonares na fase precoce são falhas na ventilação, broncoaspiração, seguidas por edema pulmonar e pneumo-hemotórax. ${ }^{12}$ Segundo Jackson e Groomers, ${ }^{45}$ atelectasia $(36,4 \%)$, pneumonia $(31,4 \%)$ e falha ventilatória $(22,6 \%)$ foram as mais prevalentes nos cinco primeiros dias de hospitalização. Outras alterações são: diminuição da força inspiratória e expiratória, do volume corrente e da capacidade residual funcional e aumento do volume residual. ${ }^{59,81,88}$ Atelectasia, distúrbios da ventilação/ perfusão, infecções, hipóxia, hipercapnia e acidose respiratória aparecem com frequência nesses casos. ${ }^{59}$ Bronquiectasias são frequentes em adultos e raros em crianças. ${ }^{100}$

O comprometimento medular, principalmente acima de T8, compromete os músculos expiratórios, diminui a efetividade da tosse e, consequentemente, altera o clearance de secreções, aumentando a suscetibilidade desses pacientes a infecções respiratórias baixas. ${ }^{12,20,88}$ Cardenas e cols., ${ }^{19}$ em uma análise multicêntrica, comprovaram a relação entre lesões medulares altas com o aumento da incidência de pneumonia.

Jackson e Groomers ${ }^{45}$ encontraram alterações respiratórias em $84 \%$ dos pacientes com lesão de $\mathrm{C} 1$ a $\mathrm{C} 4$ e em $60 \%$ nas lesões de C5 a C8. Pneumonia foi a complicação mais frequente no primeiro grupo, seguida por falhas na ventilação e atelectasias. Sessenta e cinco por cento dos pacientes com lesão de T1 a T12 apresentaram graves complicações pulmonares, mas estas foram relacionadas com traumas torácicos associados.

Alguns estudos ainda associam idade avançada, ${ }^{62}$ tetraplegia, ${ }^{52,62}$ lesões traumáticas ${ }^{61}$ e duração da lesão ${ }^{54}$ como fatores de risco para o aumento da incidência de pneumonias. Waters e cols., ${ }^{97}$ em um estudo retrospectivo, observaram prevalência de pneumonia e atelectasia em $45 \%$ dos pacientes tetraplégicos, enquanto os paraplégicos apresentaram incidência de 36\%. Nash ${ }^{69}$ correlacionou a maior disfunção imune e consequente maior predisposição a infecções com a gravidade das lesões, mostrando uma possível proporcionalidade entre ambos os fatores. Para Smith e cols., ${ }^{86}$ o nível da lesão não influencia o número de pacientes com infecção respiratória alta, provavelmente devido à etiologia viral prevalente nessa patologia.

O índice de infecções hospitalares em pacientes com TRM na fase aguda é $25,8 \%$ maior que em pacientes sem lesão medular. ${ }^{66,68}$ Além da maior predisposição a infecções respiratórias, a diminuição da capacidade inspiratória, principalmente em pacientes com comprometimento cervical alto, contribui para a formação de microatelectasias, que poderão acarretar dispneia e insuficiência respiratória, ${ }^{20,86}$ podendo simular um quadro de asma. ${ }^{20}$

Além disso, pacientes com comprometimento cervical que necessitam de respiração artificial podem desenvolver pneumonia aspirativa, principalmente se houver disfagia. A disfagia também interfere na qualidade de vida, dieta e comunicação desses pacientes. ${ }^{12,102}$

Pacientes com lesões completas acima de C3 exigem amplo suporte ventilatório controlado ou assistido-controlado. ${ }^{88}$ Os pacientes com lesões incompletas e abaixo de $\mathrm{C} 3$ necessitam de modos que incorporem a ventilação espontânea como, por exemplo, mandatória intermitente e pressão controlada. ${ }^{88}$

Pacientes com lesão cervical alta podem apresentar respiração abdominal paradoxal, ${ }^{37,88}$ ou seja, como a musculatura normal da respiração está paralisada, os músculos acessórios atuam na inspiração expandindo a caixa torácica e invertendo o diafragma e abdômen, ocorrendo o contrário na expiração. ${ }^{37,59}$ Esses pacientes apresentam um risco aumentado de parada respiratória, podendo evoluir para óbito. ${ }^{81}$ Em lesões mais baixas, a função diafragmática está preservada, mas deve-se fazer o controle respiratório para evitar fadiga do diafragma e hipoventilação. ${ }^{59}$ Claxton e cols., ${ }^{23} \mathrm{em}$ um estudo retrospectivo, avaliaram que $36 \%$ dos pacientes com TRM necessitavam de ventilação mecânica por falência respiratória. Desses, 90\% se encontravam nos três primeiros dias de hospitalização. 


\section{Alterações cardiovasculares}

O risco de alterações cardiovasculares é $200 \%$ maior em pessoas com TRM. ${ }^{47} \mathrm{O}$ coração é inervado pelo sistema simpático, de T1 a T5, e parassimpático, emergindo principalmente do bulbo. ${ }^{77}$ Lesões acima de T1 podem levar à desconexão dos centros superiores com predomínio vagal. ${ }^{75}$

O choque neurogênico, por interrupção das fibras simpáticas descendentes e predomínio da inervação parassimpática, resulta em perda do tônus vasomotor, queda da resistência vascular periférica com diminuição da pré e pós-carga, bradicardia e hipotensão arterial, que poderão resultar em insuficiência cardíaca congestiva. ${ }^{26,75,88}$

Hipotensão arterial e choque neurogênico são deletérios à medula, já que aumentam a hipoperfusão medular, perpetuando as alterações vasculares (isquemia, hemorragia, vasoespasmo, trombose e aumento da permeabilidade vascular) e inflamatórias (proliferação de citocinas, eicosanoides e leucócitos e a disfunção celular pela diminuição do trifosfato de adenosina, liberação de radicais livres, aminoácidos excitatórios e cálcio celular) secundárias ao TRM. ${ }^{29}$

Stoner e cols. ${ }^{90}$ demonstraram que pacientes com TRM têm comprometimento na função arterial em virtude da queda da dilatação mediante fluxo arterial. Comprovaram também que esse prejuízo é ainda maior nos membros inferiores. Leal e cols. ${ }^{50} \mathrm{em}$ modelo experimental com ratos, observaram que a compressão medular por 60 segundos faz aumentar a pressão arterial no momento da lesão e diminuir a frequência cardíaca, colaborando para a hipótese de descarga simpática no TRM.

Após o choque medular, o sistema nervoso simpático volta a funcionar, nessa fase de forma reflexa, sem controle cortical. Os estímulos aferentes são transmitidos pelo feixe espinotalâmico e cordões posteriores em direção ao hipotálamo. A lesão medular, cervical ou torácica alta bloqueia o estímulo, levando a uma hiperatividade simpática generalizada. ${ }^{49,77,83}$ Essa descarga simpática é responsável pela disreflexia autonômica (DA), que ocorre até três meses após o trauma, e cursa com cefaleia intensa, hipertensão arterial paroxística, midríase, piloereção, sudorese profusa, visão embaçada, bradicardia, congestão nasal e conjuntival, palidez cutânea infralesional, retração palpebral e rash cutâneo eritematoso. ${ }^{17,42,46,49,83,88,94}$ A DA ocorre da estimulação de receptores situados abaixo do nível da lesão, principalmente localizados na região urogenital. ${ }^{30,83} \mathrm{~A}$ DA aparece em mais de $90 \%$ dos pacientes com lesões acima de T6 e é mais grave quanto mais alto for o nível da lesão. ${ }^{46}$

A bradicardia diminui a perfusão dos tecidos, podendo levar à acidose e acarretando lesões secundárias para esses pacientes. ${ }^{59}$ Ocorre, em geral, da terceira a quinta semana pós-trauma. ${ }^{101} \mathrm{~A}$ bradicardia pode ocorrer em consequência da instabilidade autonômica, hipóxia, disfunção diafragmática, atelectasia, consolidação e depressão respiratória. ${ }^{77}$ Outra complicação da bradicardia, frequente em TRM agudo, é a progressão para assistolia. ${ }^{28}$ Segundo Lehman e cols. ${ }^{51}$ todos os pacientes com lesão cervical completa evoluem com bradicardia, ocorrendo assistolia em 15\% dos casos.

Entre as alterações eletrocardiográficas podem ser encontrados: batimentos ventriculares e atriais prematuros, alargamento da onda $\mathrm{T}$, onda $\mathrm{U}$, prolongamento do intervalo PR, desaparecimento de ondas P e bloqueios $\mathrm{AV}$ de segundo grau ${ }^{104} \mathrm{Na}$ radiografia simples de tórax, pode ser observada cardiomegalia. ${ }^{75}$

Além do diagnóstico clínico, a DA pode ser investigada pelo cistometrograma diferencial, no qual a pressão e o pulso do paciente são analisados mediante infusão contínua de solução salina intravesical. ${ }^{84}$

O tratamento é feito com a supressão do estímulo provocador e o controle medicamentoso da pressão arterial e sudorese supralesional compensatória. ${ }^{32}$ ADA sem tratamento pode levar a encefalopatia, crises epilépticas, acidente vascular cerebral hemorrágico, hemorragias retinianas, infarto agudo do miocárdio, insuficiência cardíaca congestiva, arritmias e óbito. ${ }^{16,83,88}$

Pacientes com lesões medulares altas, acima de T6, podem apresentar hipotensão arterial postural por interrupção simpática, apresentando um quadro de náuseas, tontura e síncope quando na posição supina. Esse sintoma é ainda mais frequente em pacientes tetraplégicos. ${ }^{17} \mathrm{Em}$ lesões de T1 a T5, acima da inervação cardíaca, pode ocorrer também inotropismo positivo e taquicardia. $^{78}$

Levi e cols. ${ }^{53}$ demonstraram que o controle e a manutenção da frequência cardíaca, pressão arterial, pressão da artéria pulmonar, ritmo cardíaco, resistência vascular sistêmica e pulmonar e a oxigenação dentro dos padrões melhoram o prognóstico e o quadro neurológico desses pacientes.

\section{Alterações urogenitais}

A bexiga é controlada por mecanismos voluntários e involuntários. O esfíncter uretral externo tem inervação somática proveniente da região sacral que permite o controle voluntário da micção. ${ }^{14}$ Em sua maioria, pacientes com TRM perdem o controle do esfíncter externo. Sem o relaxamento do esfíncter concomitante à contração vesical, há aumento na pressão intravesical, levando à distensão vesical e superestiramento da bexiga e do músculo detrusor, podendo afetar, inclusive, 
a taxa de filtração glomerular renal. ${ }^{14,17}$ Nesses casos, a retenção urinária é o resultado imediato da lesão medular. ${ }^{17}$ A dilatação ureteral, por insuficiência na propulsão urinária, pode levar também à hidronefrose. ${ }^{14}$

$\mathrm{O}$ cateterismo vesical intermitente deve ser feito a cada quatro ou seis horas, de acordo com o balanço hídrico do paciente, ou por meio de sonda de demora, que deve ser aberta a cada três ou quatro horas, sendo trocada semanalmente. ${ }^{17,60,87}$ A cateterização intermitente $(\mathrm{CI})$ feita pelo próprio paciente com adequadas técnicas de higiene não aumenta o risco de infecção, pelo contrário, diminui o risco de infecções cruzadas entre pacientes. ${ }^{76,104}$ Entretanto, a CI pode levar à formação de fístulas, estreitamentos ureterais, uretrites e epididimorquites. ${ }^{103}$

Biering-Sorensen e cols., ${ }^{13}$ analisando 77 pacientes com TRM após cinco anos de CI, observaram que $81 \%$ dos pacientes já tinham sido tratados de infecções do trato urinário (ITU) pelo menos uma vez, 22,2\% dos pacientes tiveram de dois a três episódios de ITU por ano e $12 \%$, quatro episódios ou mais.

Wyndaele, ${ }^{104}$ em revisão da literatura, relata prevalência de Escherichia coli, Proteus, Citrobacter, Pseudomonas sp., Klebsiella sp., Staphylococcus aureus e faecalis em pacientes com CI de curta e longa duração, além de Acinetobacter e Streptococcus faecalis em pacientes com CI prolongada. Bakke ${ }^{7}$ encontrou prevalência de Escherichia coli em pacientes com CI. Noventa e três por cento dos pacientes com Escherichia coli na periuretra apresentam bacteriúria. ${ }^{82}$

O uso de sonda vesical de demora predispõe a infecções como cistite, pielonefrite, litíase vesical e fístulas uretrocutâneas. ${ }^{59}$ A ITU é a complicação mais frequente em pacientes com TRM agudo. ${ }^{96,103}$ Segundo Cabrera e cols., ${ }^{18}$ a maior variável de risco para ITU foi o tempo de hospitalização prolongado.

A bacteriúria, presente em grande parte dos pacientes com TRM, geralmente é assintomática, mas pode causar alterações em longo prazo como cálculos renais, pielonefrites, falência renal, bacteremia, entre outros. ${ }^{76}$ Para Penders e cols.,${ }^{76}$ a Klebsiella pneumoniae mostrou-se o patógeno mais prevalente em paciente com TRM. Pacientes tetraplégicos com cateterização prolongada tendem a apresentar maior resistência bacteriana que outros pacientes com uso crônico de sondas. ${ }^{70}$ A terapêutica empírica para ITU em pacientes com TRM é indicada na presença de sintomas clínicos logo após a coleta de amostra para realização da urocultura. ${ }^{70,76} \mathrm{~A}$ antibioticoterapia deve ser ajustada após o resultado desse exame. O tratamento de primeira escolha é feito com cefuroxime e fluoroquinolona. ${ }^{76}$

Galloway e cols. ${ }^{35}$ avaliaram a efetividade da antibioticoprofilaxia para pacientes com TRM. O risco de resistência bacteriana, a dificuldade no tratamento de emergência para patógenos resistentes, os efeitos colaterais dessas medicações, além dos gastos e riscos de infecções cruzadas mais resistentes entre os pacientes hospitalizados, mostraram que o risco sobrepõe o benefício do uso dessas medicações. Wyndaele e De Taeye ${ }^{104}$ relataram choque séptico em $18,26 \%$ dos pacientes com TRM durante internação hospitalar. O uso de sonda vesical de demora predispõe a essa complicação. . $^{8,104}$

Em pacientes com lesão sacral, pode haver dificuldade na contração do esfíncter, levando a um quadro de incontinência urinária. ${ }^{14}$ Alterações motoras, sensitivas e autonômicas podem levar também à disfunção sexual em pacientes com TRM. Esta pode ser primária, quando por alterações orgânicas, ou secundária, quando ocorre por mudanças psicossociais, e merece atenção dos profissionais de saúde. $^{91}$

Infecções genitais podem levar à infertilidade nos pacientes com TRM. ${ }^{103}$ Allas e cols. ${ }^{2}$ avaliaram a função sexual em homens paraplégicos com CI. Foram realizados dois espermogramas, um antes e outro após um episódio de epididimite, e foi encontrado aumento da azoospermia de $7 \%$ para $50 \%$ após epididimite. No entanto, para esvaziamento de bexiga neurogênica, pacientes com CI apresentam taxas de fertilidade maiores que pacientes com sonda vesical de demora. ${ }^{73,80}$

\section{Alterações gastrintestinais}

Cerca de 1,5 milhão de pessoas, nos Estados Unidos, apresentam lesão medular. Dessas, aproximadamente 400 mil apresentam sintomas gastrintestinais crônicos que são ainda mais frequentes com a progressão da lesão. ${ }^{71,89} \mathrm{Na}$ fase crônica da doença, pesquisas mostram que um terço dos pacientes relata que as alterações colorretais alteram mais a qualidade de vida que as alterações geniturinárias. ${ }^{38,43}$ A continência fecal é mantida pelo tônus e atividade reflexa dos esfíncteres anais internos e externos e pelos músculos do assoalho pélvico. ${ }^{55}$

O esfíncter anal externo, inervado pelo nervo pudendo, que surge de S2 a S4, é responsável pelo controle voluntário da defecação. Lesões acima de S2S4 podem levar à perda do controle voluntário sobre a defecação ou à contração mais vigorosa do esfíncter externo anal (EEA). ${ }^{17,71}$ Lesões acima de S2-S4 também comprometem o nervo pélvico parassimpático, que pode ocasionar hipotonia retal, perda do reflexo retocólico com atraso no esvaziamento intestinal ${ }^{71}$. Essas alterações podem trazer constipação, empachamento, distensão abdominal e íleo paralítico ${ }^{71}$. Lesões acima de T5 alteram a coordenação dos movimentos peristálticos entre o antro e o duodeno, causando atraso também no esvaziamento gástrico. ${ }^{31}$ 
Em lesões completas ou parciais da cauda equina, o EEA e a musculatura pélvica se tornam flácidos e perdem a capacidade reflexa de responder ao aumento da pressão intra-abdominal. A perda do controle parassimpático e da inervação reflexa do esfíncter anal interno reduz o relaxamento do tônus anal e leva à incontinência fecal.

Em estudo comparativo, foi analisada a prevalência de incontinência fecal em pacientes com TRM com controles do mesmo sexo e gênero. A incontinência fecal foi maior em pacientes com TRM e ainda mais frequente em pacientes com lesão medular completa quando comparados com pacientes com lesão parcial. ${ }^{56}$ A incontinência fecal afetou a qualidade de vida de $62 \%$ dos pacientes comparados com apenas $8 \%$ no grupo controle. ${ }^{56} \mathrm{O}$ uso de laxantes $(39 \%$ contra $4 \%$ ), assim como hemorroidectomias ( $9 \%$ contra $1,5 \%$ ), foi mais frequente nos grupos de pacientes com TRM quando comparados com os controles. A hemorroidectomia foi ainda mais frequente em pacientes que necessitavam de evacuações manuais. ${ }^{56}$

O estímulo endógeno e exógeno de esteroides, assim como a hiperestimulação vagal, predispõe à formação de úlceras gástricas por estresse, chamadas de úlceras de Cushing, que podem acarretar episódios de sangramento digestivo alto com hematêmese e melena. ${ }^{59,88}$ Esse risco é ainda mais elevado em pacientes em ventilação mecânica e em uso de altas doses de corticosteroides. ${ }^{88}$

Uma complicação tardia, frequente em pacientes com TRM, é a colelitíase. , $, 5,67,79$ Uma das teorias que tentam explicar a etiologia dos cálculos biliares em pacientes com TRM retrata alteração no ciclo intestinal dos sais biliares em pacientes cujo trânsito intestinal encontra-se mais lentificado. Em estudo comparativo, Rotter e Larraín ${ }^{79}$ encontraram prevalência de colelitíase em $25 \%$ dos pacientes com TRM contra 9\% no grupo controle. Não foi encontrada diferença estatisticamente significante quando analisados: nível neurológico da lesão, duração da lesão, idade e índice de massa corporal nesses pacientes. ${ }^{67,79}$ Apstein e cols. ${ }^{5}$ encontraram maior prevalência de colelitíase em pacientes com lesão acima de T10 devido ao comprometimento do sistema simpático. Moonka e cols. ${ }^{67}$ relataram maior incidência de colelitíase em pacientes com lesão medular completa.

\section{Trombose venosa profunda}

Outra complicação grave em paciente com TRM é a trombose venosa profunda (TVP). Entre todas as admissões hospitalares, os pacientes com TRM agudo apresentam o maior risco de desenvolver TVP. ${ }^{6,36} \mathrm{~A}$ incidência de TVP nos primeiros três meses de paralisia por lesão medular é de $38 \%,{ }^{1}$ com pico de incidência entre 7 e 10 dias. $^{64} \mathrm{~A}$ incidência pode variar de $9 \%$ a $90 \%$, dependendo dos métodos diagnósticos empregados nos estudos. ${ }^{9,10,21,95} \mathrm{O}$ risco de TVP é maior em pacientes com lesões medulares completas $(81 \%)$ que incompletas $(8 \%)$, torácicas que cervicais, principalmente nos primeiros três meses após a lesão. ${ }^{36,59,98,99}$

A diminuição do tônus vasomotor, do retorno venoso e da contração muscular, a hipercoagulabilidade, a imobilização e o dano direto no sistema venoso profundo predispõem à TVP os pacientes com TRM na fase aguda. ${ }^{1,59}$ A TVP pode levar à embolia pulmonar, importante causa de morte em pacientes com TRM. ${ }^{22,27,81,85,106}$ A frequência de tromboembolismo pulmonar (TEP) nos primeiros três meses após a lesão medular é de aproximadamente $5 \%$, sendo o risco maior nas duas primeiras semanas após a lesão. ${ }^{3}$

Para Green e cols., ${ }^{41}$ o TEP se mostrou mais frequente em pacientes com tetraplegia, paralisia completa, alto nível da lesão neurológica, alto índice de massa corporal e ausência de espasticidade.

As evidências clínicas de TVP são raras. Aito e cols. ${ }^{1}$ encontraram evidências clínicas em $9 \%$ dos casos em cerca de $35 \%$ do total de pacientes com TVP. Nesse estudo, em apenas $2 \%$ dos pacientes admitidos durante as primeiras 72 horas do trauma foi detectada TVP. Nos pacientes admitidos entre 8 e 28 dias após a lesão, 26\% dos casos tiveram TVP. As medidas profiláticas contra a TVP eram empregadas logo após a internação.

Para Yelnik e cols., ${ }^{105} 40 \%$ dos pacientes com venografia evidenciando trombose apresentavam sintomatologia clínica para TVP. Nesse estudo, TEP foi a causa de morte em 1,5\% dos casos.

Pacientes com lesão medular necessitam de profilaxia de rotina para TVP. O tratamento é feito com heparina de baixo peso molecular, warfarin, baixa dose de heparina não fracionada, além de medidas não medicamentosas como mobilização precoce, compressão pneumática dos membros inferiores e uso de meias elásticas. ${ }^{1,3}$ Apesar de cuidados profiláticos adequados, cerca de $4,5 \%$ dos pacientes com TRM têm como causa do óbito a TEP. ${ }^{39,40}$

\section{Úlceras de pressão}

Pacientes imobilizados, com alteração da sensibilidade, irrigação sanguínea, elasticidade e turgor da pele, têm tendência a desenvolver úlceras de pressão. ${ }^{17,59}$ Os locais mais acometidos estão sobre proeminências ósseas ou próteses..$^{59,72}$

De acordo com a profundidade da lesão, elas podem ser classificadas em grau I (eritema sem lesões de pele), 
grau II (comprometimento de epiderme e derme), grau III (lesão até o tecido celular subcutâneo) e grau IV (lesão extensa com necrose tecidual e dano aos tecidos profundos). ${ }^{59}$

Devem ser realizadas mudanças de decúbito, de preferência a cada duas horas, fazendo cuidados locais na pele para evitar a formação de úlceras de pressão.

\section{Referências}

1. Aito S, Pieri A, D'Andrea M, Marcelli F, Cominelli E. Primary prevention of deep venous thrombosis and pulmonary embolism in acute spinal cord injured patients. Spinal Cord. 2002;40:300-3.

2. Allas $T$, Colleu $D$, Le Lannon D. Fonction génitale chez I'homme praplégique. Aspects immunologiques. Presse Med. 1986;29:2119.

3. Anderson Jr FA, Spencer FA. Four topics in venous thromboembolism. Risk factors for venous thromboembolism. Circulation. 2003;107:1-9.

4. Apstein MD, Dalecki-Chipperfield K. Spinal cord injury is a risk factor for gallstone disease. Gastroenterology. 1987;92:966-8.

5. Apstein MD, George B, Tchakarova B. Spinal cord injury is a risk factor for cholesterol gallstone disease. Gastroenterology. 1991;100:715.

6. Attia J, Ray JG, Cook DJ, Douketis J, Ginsberg J, Geerts W. Deep vein thrombosis and its prevention in critically ill adults. Arch Intern Med. 2001;161:1268-79.

7. Bakke A. Clean intermittent catheterization-physical and psychological complications. Scan J Urol Nephrol (Suppl). 1993;150:1-69.

8. Barkin M, Dolfin D, Herschorn S, Bharatwal N, Comisarow R. The urological care of the spinal cord injury patient. J Urol. 1983;129:335-9.

9. Barrellier MT, Jouen E, Creveuil C. Discrepancies between the results of phlebography and Doppler ultrasonography in the diagnosis of asymptotic venous thrombosis after total hip prosthesis. False negatives of phlebography or false positives of Doppler ultrasonography. J Mal Vasc. 1998:23:183-90.

10. Barrellier MT, Lenzin B, Landy S, Le Hello C. Prevalance de la thrombose veineuse diagnostique par echographiedoppler des membres inferieurs dans la suspicion d'embolie pulmonaire et dans l'embolie pulmonaire confirmee. J Mal Vasc. 2001;26:23-30.

11. Bellamy R, Pittys FW, Stauffere S. Respiratory complications of traumatic quadriplegia. J Neurosurg. 1973;34:596-600.

12. Berlly M, Shem K. Respiratory management during the first five days after spinal cord injury Spinal Cord Med. 2007;30:309-18.

13. Biering-Sorensen F, Nielans HM, Dorflinger T, Sorensen B. Urological situation five years after spinal cord injury. Scand J Urol Nephrol. 1999;33:157-61.

14. Bodner DR. Evidence-based management of the neurogenic bladder: a new clinical practice guideline. J Spinal Cord Med. 2006;29:479.

15. Botelho RV, Abgussen CMB, Machado GCFP, Elias AJR, Benedito-Silva AA, Bittencourt LRA, et al. Epidemiologia do trauma raquimedular cervical na zona norte da cidade de São Paulo. Arq Bras Neurocir (Sao Paulo). 2001;20:64-76.
16. Braddon RL, Rocco JF. Autonomic disreflexia: a survey of current treatment. Arch Phys Med Rehabil. 1991;70:234-41.

17. Bruni DS, Strazzieri KC, Gumieiro MN, Giovanazzi R, Sá VG, Faro ACM. Aspectos fisiopatológicos e assistenciais de enfermagem na reabilitação da pessoa com lesão medular. Rev Esc Enferm USP. 2004;38:71-9.

18. Cabrera RH, Arrazola AL, Alcaide MJV, Arjona MF, Calero JR. Analytic epidemiology of clinical urinary tract infection in spinal cord injury. Europ J Epidemiol. 1994;10:23-7.

19. Cardenas DD, Hoffman JM, Kirshblum S, Mckinley W. Etiology and incidence of rehospitalization after traumatic spinal cord injury: a multicenter analysis. Arch Phys Med Rehabil. 2004;85:1757-63.

20. Cardozo CP. Respiratory complications of spinal cord injury. Spinal Cord Med. 2007;30:307-8.

21. Casas ER, Sanchez MP, Arias CR, Masip JP. Prophylaxis of venous thrombosis and pulmonary embolism in patients with acute traumatic spinal cord lesions. Paraplegia. 1977; 15:209-14.

22. Chu DA, Ahln MD, Ragnarsson KT. Deep venous trombosis; Diagnosis in spinal cord injured patients. Arch Phys Med Rehab. 1985;66:365-8.

23. Claxton R, Wong D, Chung F, Fehlings M. Predictors of hospital mortality and mechanical ventilation in patients with cervical spinal cord injury. Can J Anaesthesiol. 1998:45:144-9.

24. Dantas Filho VP. Trauma raquimedular. In: Lopes AC, editor. Tratado de clínica médica. São Paulo: Roca; 2006. v. 2 , p. $2305-10$.

25. Daverat P, Gagnon M, Dartigues JF, Mazaux JM, Barat M. Initial factors predicting survival in patients with a spinal cord injury. J Neurol Neurosurg Psychiatry. 1989;52:403-6.

26. Defino HLA. Trauma raquimedular. Medicina. Simpósio: Trauma II. 1999;32:388-400.

27. Devivo MJ, Krause JS, Lammertse DP. Recent trends in mortality and causes of death among persons with spinal cord injury. Arch Phys Med Rehabil. 1999;80:1411-9.

28. Dixit $\mathrm{S}$. Bradycardia associated with high cervical spinal cord injury. Surg Neurol. 1995;43:514.

29. Dumont RJ, Okonkwo DO, Verma S, Hurlbert RJ, Boulos PT, Ellegala DB, et al. Acute spinal cord injury. Part I: Pathophysiologic mechanisms. Clin Neuropharmacol. 2001;24:254-64.

30. Erickson RP. Autonomic hyperreflexia: pathophysiology and medical management. Arch Phys Med Rehabil. 1980;61:431-40.

31. Fealey AD, Szurszewski JH, Merritt JL, Dimagno EP. Effect of traumatic spinal cord transection on human upper gastrointestinal motility and gastric emptying. Gastroenterology. 1984;87:69-75.

32. Feustel D. Autonomic hyperreflexia. Am J Nurs. 1976;76: 228-30.

33. Fine PR, Kuhlemeier KV, De Vivo WJ, Stover SL. Spinal cord injury: an epidemiology perspective. Paraplegia. 1979;17:237-50.

34. Frankel HL, Hancock DO, Hyslop G, Melzak J, Michaelis $\mathrm{LS}$, Uogar GH, et al. The value of postural reduction in the initial management of closed injuries of spine with paraplegia and tetraplegia. Paraplegia. 1969;7:179-92.

35. Galloway A, Green HT, Windsor JJ, Menon KK, Gardner BP, Krishnan KR. Serial concentrations of creatinine protein as an indicator of urinary tract infection in patients with spinal injury. J Clin Pathol. 1986;39:851-5.

36. Geerts WH, Heit JA, Clagett GP, Pineo GF, Colwell $\mathrm{CW}$, Anderson Jr FA, et al. Prevention of venous thromboembolism. Chest. 2001;119:132-75. 
37. Giffin JP, Grush K. Spinal cord injury treatment and the anesthesiologist. In: Lee BY, Ostrander LE, Cochran GVB, editors. The spinal cord injury patient: comprehensive management. Philadelphia: WB Saunders; 1991. p. 308-30

38. Glickman S, Kamm MA. Bowel dysfunction in spinal cord injury patients. Lancet. 1996;347:1651-3.

39. Green D, Chen D, Chmiel JS, Olsen NK, Berkowitz M, Novick $A$, et al. Prevention of thromboembolism in spinal cord injury: role of low molecular weight heparin. Arch Phys Med Rehabil. 1994;75:290-2.

40. Green D, Lee MY, Lim AC, Chmiel JS, Vetter M, Pan G, Chen D, et al. Prevention of thromboembolism after spinal cord injury using low-molecular weight heparin. Ann Intern Med. 1990;113:571-4.

41. Green D, Twardowski P, Wei R, Rademaker AW. Fatal pulmonary embolism in spinal cord injury. Chest. 1994;105:853-5.

42. Guttmann L, Whitteridge D. Effects of bladder distension on autonomic mechanisms after spinal cord injuries. Brain. 1947;70:361-404

43. Han TR, Kim JH, Kwon BS. Chronic gastrointestinal problems and bowel dysfunction in patients with spinal cord injury. Spinal Cord. 1998;36:485-90.

44. Holly LT, Kelly DF, Counelis GJ, Blinman T, Mcarthur DL, Cryer HG. Cervical spine trauma associated with moderate and severe head injury: incidence, risk factors, and injury characteristics. J Neurosurg. 2002;96:285-91.

45. Jackson AB, Groomers TE. Incidence of respiratory complications following SCl. Arch Phys Med Rehabil. 1994;75:270-5.

46. Karlsson AK. Autonomic dysreflexia. Spinal Cord. 1999;37:383-91.

47. Kocina P. Body composition of spinal cord injured adults. Sports Med. 1997;23:48-60

48. Kraus JF, Franti CE, Riggins RS, Richards D, Borhani NO. Incidence of traumatic spinal cord lesions. J Chron Dis. 1975;28:471-92

49. Kurnick NB. Autonomic hyperreflexia and its control in patients with spinal cord lesions. Ann Intern Med 1956;44:678-86.

50. Leal Filho MB, Morandin RC, Almeida AR, Cambiucci EC, Metze K, Borges G, et al. Hemodynamic parameters and neurogenic pulmonary edema following spinal cord injury: an experimental model. Arq Neuro-Psiquiatr (Sao Paulo). 2005;63:990-6.

51. Lehman K, Lane J, Piepmeir J, Batsford W. Cardiovascular abnormalities accompanying acute spinal cord injury in humans: incidence, time course and severity. J Am Coll Cardiol. 1987;10:46-52.

52. Lemons VR, Wagner FC Jr. Respiratory complications after cervical spinal cord injury. Spine. 1994;19:2315-20.

53. Levi L, Wolf A, Belzberg $\mathrm{H}$. Hemodinamic parameters in patients with acute cervical cord trauma: description, intervention, and prediction of outcome. Neurosurgery. 1993;33:1007-17.

54. Levi R, Hultling C, Seiger A. The Stockholm Spinal Cord Injury Study: 2. Associations between clinical patient characteristics and post-acute medical problems. Paraplegia. 1995;33:585-94.

55. Lynch AC, Antony A, Dobbs BR, Frizelle FA. Bowel dysfunction following spinal cord injury. Spinal Cord. 2001;39:193-203.

56. Lynch AC, Wong C, Antony A, Dobbs BR, Frizelle FA Bowel dysfunction following spinal cord injury: a description of bowel function in a spinal cord-injured population and comparison with age and gender matched controls. Spinal Cord. 2000;38:717-23

57. Marshall LF, Knowltons, Garfin SR, Klauber MR, Elsenberg HM, Kopaniky D, et al. Deterioration following spinal cord injury: a multicenter study. J Neurosurg. 1987;66:440-4.

58. McBridge D. Spinal cord injury syndromes. In: Greenberg J, editor. Handbook of head and spinal trauma. New York: Macel Dekker; 1993. p. 393.

59. McBridge D, Rodts GE. Intensive care of patients with spinal trauma. Neurosurg Clinic North Am. 1994;30:755-66.

60. McGuire EJ, Diddel G, Wagner Jr F. Balanced bladder function in spinal cord injury patients. J Urol. 1977;118:626-8.

61. McKinley W, Cifu D, Keyser-Marcus L, Wilson K. Comparison of rehabilitation outcomes in violent vs non-violent traumatic SCI. J Spinal Cord Med. 1998;21:32-6.

62. McKinley WO, Jackson AB, Cardenas DD, Devivo MJ. Long-term medical complications after traumatic spinal cord injury: a regional model systems analysis. Arch Phys Med Rehabil. 1999;80:1402-10.

63. McMichan JC, Michel L, Westbrook PR. Pulmonary dysfunction following traumatic quadriplegia. Recognition, prevention, and treatment. JAMA. 1980;243:528-31.

64. Merli GJ. Management of deep venous trombosis in spinal cord injury. Chest. 1992;102:652-7.

65. Michael DB, Guyot DR, Darmody WR. Coincidence of head and cervical spine injury. J Neurotrauma. 1989;6:177-89.

66. Montgomerie JZ. Infections in patients with spinal cord injuries. Clin Infect Dis. 1997;25:1285-92.

67. Moonka R, Stiens SA, Resnick WJ, Mcdonald JM, Eubank WB, Dominitz JA, et al. The prevalence and natural history of gallstones in spinal cord injury. J Am Coll Surg. 1999;189:274-81.

68. Mylotte JM, Graham R, Kahler L, Young L, Goodnough S. Epidemiology of nosocomial infection and resistant organisms among patients admitted for the first time to an acute rehabilitation unit. Clin Infect Dis. 2000;30:425-32.

69. Nash MS. Immune dysfunction and illness susceptibility after spinal cord injury: an overview of probable causes, likely consequences, and potential treatments. J Spinal Cord Med. 2000;23:109-10.

70. Nicolle LE. The chronic indwelling catheter and urinary infection in long-term-care facility residents. Infect Control Hasp Epidemiol. 2001;22:316-21.

71. Nino-Murcia M, Friedland GW. Functional abnormalities of the gastrointestinal tract in patients with spinal cord injuries: evaluation with imaging procedures. AJA. 1992;158:279-81.

72. Nsenis JE, Sarmiento A. The pathophysiology and management of pressure sores. Orthop Rev. 1973;2:25-34.

73. OhI DA, Denil J, Fitzgerald-Shelton K, McCabe M, McGuire EJ, Randolph JF. Fertility of spinal cord injured males: effect of genitourinary infection and bladder management on results of electroejaculation. J Am Paraplegia Soc. 1992:15:53-9.

74. Papia G, McLellan BA, El-Helou P, Louie M, Rachlis A, Szalai $\mathrm{JP}$, et al. Infection in hospitalized trauma patients: incidence, risk factors, and complications. J Trauma. 1998;47:923-7.

75. Payno PB, Bertol CL, Álvarez JM. Alteraciones cardiovasculares en los lesionados medulares cervicales. Rev Clín Esp. 1991;188.

76. Penders J, Huylenbroeck AAY, Everaert K, Van Laere M, Verschraegen GLC. Urinary infections in patients with spinal cord injury. Spinal Cord. 2003;41:549-52.

77. Pereira CU, Sousa ACS, Lima MA, Santos PCF, Santos EAS. Alterações eletrocardiográficas em pacientes com traumatismo da coluna cervical. Arq Bras Neurocir (São Paulo). 2006;25:60-5. 
78. Raeder JC, Gisvold SE. Perioperative autonomic hyperreflexia in high spinal cord lesions: a case report. Acta Anaesthesiol Scand. 1986;30:672-3.

79. Rotter KP, Larraín CG. Gallstones in spinal cord injury (SCI): a late medical complication? Spinal Cord. 2003;41:105-8.

80. Rutkowski SB, Middleton JW, Truman G, Hagen DL, Ryan JP. The influence of bladder management on fertility in spinal cord injured males. Paraplegia. 1995;33:263-6.

81. Schimitt J, Midha M, Mckenzie N. Medical complications of spinal cord disease. In: Woolsey RM, Young RR, editors. Neurologic clinics. Philadelphia: WB Saunders; 1991. p. 779-95.

82. Schlager TA, Hendley JO, Wilson RA, Simon V, Whittam TS. Correlation of periurethral bacterial flora with bacteriuria and urinary tract infection in children with neurogenic bladder receiving intermittent catheterization. Clin Infect Dis. 1999;28:346-50.

83. Setton ARF, Pereira CU, Santos JL. Hiperreflexia autonômica simpática. J Bras Neurocirurg. 1995;6:49-54.

84. Shea JD, Gioffre R, Carrion H, Small MP. Autonomic hyperreflexia in spinal cord injury. South Med J. 1973;66: 869-72.

85. Simon RJ. Thrombosis of the inferior vena cava in a patient with spinal cord injury. Arch Phys Med Rehab. 1987:68:178-9.

86. Smith BM, Charlesnika TE, Kurichi JE, Weaver FM, Patel N, Burns SP. Acute respiratory tract infection visits of veterans with spinal cord injuries and disorders: rates, trends, and risk factors. J Spinal Cord Med. 2007;30:355-61.

87. Sperling KB. Intermittent catheterization to obtain catheterfree bladder in spinal cord injury. Arch Phys Med Rehabil. 1978;59:4-8

88. Stevens RD, Bhardwaj A, Kirsch JR, Mirski MA. Critical care and perioperative management in traumatic spinal cord injury. J Neurosurg Anesthesiol. 2003;15:215-29.

89. Stone JM, Nino-Murcia M, Wolfe VA, Perkash I. Chronic gastrointestinal problems in spinal cord injury patients: a prospective analysis. Am J Gastroenterol. 1990;85:1114-9.

90. Stoner L, Sabatier M, Vanhhiel L, Groves D, Ripley D, Palardy G, Mccully K. Upper vs lower extremity arterial function after spinal cord injury. J Spinal Cord Med. 2006;29:138-46.

91. Suaid HJ, Rocha JN, Martins ACP, Cologna AJ, Suaid $\mathrm{CA}$, Ribeiro AGB, et al. Abordagem pelo urologista da sexualidade no lesado raquimedular. Acta Cir Bras. 2002;17:41-3.

92. Surkin J, Gilbert BJ, Harkey HL, Sniezek J, Currier M. Spinal cord injury in Mississippi: findings and evaluation, 1992-1994. Spine. 2000;25:716-21.
93. Tator $\mathrm{CH}$. Classification of spinal cord injury based on neurological presentation. In: Narayan RK, Wilberg JE, Povilishock, JT, editors. Neurotrauma. New York: Mc GrawHill; 1996. p. 1053-73.

94. Thompson CE, Witham AC. Paroxysmal hypertension in spinal cord injuries. N Engl J Med. 1948;239:291-4.

95. Waring WP, Karunas RS. Acute spinal cord injuries and the incidence of clinically occurring thromboembolic disease. Paraplegia. 1991;29:8-16.

96. Warren JM, Muncie HL, Bergquist EJ, Hoopes JM. Sequelae and management of urinary infection in the patient requiring chronic catheterization. J Urol. 1981;125:1-8.

97. Waters R, Meyer P, Adkins R, Felton D. Emergency, acute, and surgical management of spine trauma. Arch Phys Med Rehabil. 1999;80:1383-90.

98. Watson W. Venous thrombosis and pulmonary embolism in spinal cord injury patients. Paraplegia. 1978;16:113-21.

99. Weingarden SI. Deep venous thrombosis in spinal cord injury: overview of the problem. Chest.1992102:636-9.

100. Wilberg JE. Intensive care management of pediatric spinal cord injury In: Andrews BT, Hammer GT, editors. Pediatric neurosurgical intensive care. Philadelphia: Saunders; 1997. p. 139-45.

101. Wineinger MA, Basford JR. Autonomic dysreflexia due to medication: misadventure in the use of an isometheptene combination to treat migraine. Arch Phys Med Rehabil. 1985;66:645-6.

102. Wolf C, Meiners TH. Dysphagia in patients with acute cervical spinal cord injury. Spinal Cord. 2003;41:347-53.

103. Wyndaele JJ. Complications of intermittent catheterization: their prevention and treatment. Spinal Cord. 2002;40: 536-41.

104. Wyndaele JJ, De Taeye N. Early intermittent selfcatheterization after spinal cord injury. Paraplegia. 1990;28:76-80.

105. Yelnik A, Dizien O, Bussel B, Schouman-Claeys E, Frija G, Pannier S, et al. Systematic lower limb phlebography in acute spinal cord injury in 147 patients. Paraplegia. 1991;29:253-60.

106. Yeo JD, Walsh J, Rutkowski S, Soden R, Craven M, Middleton J. Mortality following spinal cord injury. Spinal Cord. 1998;36:329-36.

Endereço para correspondência

Carlos Umberto Pereira

Av. Augusto Maynard, 245/404

49015-380 - Aracaju, SE

E-mail: umberto@infonet.com.br 